\title{
Perfil clínico e epidemiológico de pacientes com lesão por pressão: estudo comparativo entre hospital público e privado
}

\author{
Clinical and epidemiological profile of patients with pressure injuries: a comparative study between \\ public and private hospitals \\ Perfil clínico y epidemiológico de pacientes con lesiones por presión: un estudio comparativo entre \\ hospitales públicos y privados
}

Iris Waleska Rodrigues de Melo Bastos

ORCID: https://orcid.org/0000-0001-8385-9053 Universidade Estadual de Ciências da Saúde de Alagoas, Brasil E-mail: waleska_bastos@hotmail.com Lara Freire de Menezes Costa ORCID: https://orcid.org/0000-0002-1959-6436 Universidade Estadual de Ciências da Saúde de Alagoas, Brasil E-mail: larafreiremenzes@hotmail.com

Meryanne Fernandes de Aguiar Ferreira ORCID: https://orcid.org/0000-0003-3051-729X Universidade Estadual de Ciências da Saúde de Alagoas, Brasil E-mail: meryannef@gmail.com

Lucyo Wagner Torres de Carvalho ORCID: https://orcid.org/0000-0002-7990-0209 Universidade Estadual de Ciências da Saúde de Alagoas, Brasil E-mail:1wtc@hotmail.com

Sandra Adriana Zimpel

ORCID: https://orcid.org/0000-0002-6551-9888 Universidade Estadual de Ciências da Saúde de Alagoas, Brasil E-mail: sandrazimpel@uol.com.br

Priscila Godoy Januário Martins Alves ORCID: https://orcid.org/0000-0002-5992-2443 Universidade do Estado da Bahia, Brasil E-mail: pgjmalves@uneb.br

Rodrigo José Nunes Calumby

ORCID: https://orcid.org/0000-0002-2313-5552 Universidade Federal de Alagoas, Brasil E-mail: rjncbiomed@hotmail.com

Juliane Cabral Silva

ORCID: https://orcid.org/0000-0003-3098-1885 Universidade Estadual de Ciências da Saúde de Alagoas, Brasil Centro Universitário CESMAC, Brasil E-mail: larbacjuliane@gmail.com

\begin{abstract}
Resumo
A lesão por pressão (LP) acontece quando um tecido é comprimido por uma proeminência óssea e uma superfície. Sua presença pode agravar o estado de saúde do paciente, gerando dor e comprometimento sistêmico. As altas taxas de incidência, prevalência, mortalidade e de custos provenientes dessa condição, representam um grave problema de saúde pública. Este estudo teve por objetivo conhecer o perfil clínico e epidemiológico de pacientes que adquiriram a LP durante o processo hospitalar, comparando características entre o hospital público e o privado. Trata-se de um estudo analítico, comparativo, de abordagem quantitativa, realizado pela análise de prontuários. O perfil clínico geral dos pacientes que adquiriram LP no ambiente hospitalar foi: $50 \%$ feminino e $50 \%$ masculino; idoso (62\%); com período de internação curto <1 mês (64\%), que receberam atendimento multidisciplinar (97\%) e fisioterapêutico (86\%) e vieram a óbito $(68 \%)$. A realidade sociodemográfica e clínica dos pacientes foram semelhantes, o que demonstra que os fatores de risco não são relacionados ao tipo de assistência pública ou privada, exceto em relação a faixa etária e a doença associada. Ressaltamos a alta incidência de óbito encontrada na população estudada, o que demonstra a necessidade de ampliar o cuidado preventivo.
\end{abstract}

Palavras-chave: Fatores de risco; Lesão por pressão; Hospitais. 


\begin{abstract}
Pressure injury (LP) occurs when a tissue is compressed by a bone prominence in contact with a surface. Their presence may aggravate the patient's state of health, generating pain and systemic impairment. The high rates of incidence, prevalence, mortality and costs from this condition, represent a serious public health problem. This study aimed to know the clinical and epidemiological profile of patients who acquired the LP during the hospitalization period, comparing the characteristics between the public and private hospitals. This is an analytical, comparative study with a quantitative approach, carried out by analyzing medical records, of adult patients who acquired LP during hospitalization in public and private hospitals. The general clinical profile of patients who acquired pressure injury in the hospital setting was: $50 \%$ female and 50\% male; elderly (62\%); with short hospital stay <1 month (64\%), who received multidisciplinary care $(97 \%)$ and physiotherapy $(86 \%)$ and died (68\%). The socio-demographic and clinical reality of the patients who acquired LP in both hospitals were similar, which shows that the risk factors are not related to the type of public or private assistance, except in relation to the age group and associated disease. We emphasize the high incidence of death found in the studied population, which demonstrates the necessity to extend the preventive.
\end{abstract}

Keywords: Risk factors; Pressure injury; Hospitals.

\title{
Resumen
}

La lesión por presión (LP) ocurre cuando un tejido es comprimido por una prominencia ósea y una superficie. Su presencia puede empeorar el estado de salud del paciente, generando dolor y deterioro sistémico. Las altas tasas de incidencia, prevalencia, mortalidad y costos derivados de esta condición, representan un grave problema de salud pública. Este estudio tuvo como objetivo conocer el perfil clínico y epidemiológico de los pacientes que adquirieron LP durante el proceso hospitalario, comparando características entre hospitales públicos y privados. Se trata de un estudio analítico, comparativo con enfoque cuantitativo, realizado mediante el análisis de historias clínicas. El perfil clínico general de los pacientes que adquirieron LP en el ámbito hospitalario fue: 50\% mujeres y 50\% hombres; ancianos (62\%); con una estancia hospitalaria corta <1 mes (64\%), que recibió atención multidisciplinar (97\%) y fisioterapia $(86 \%)$ y falleció (68\%). La realidad sociodemográfica y clínica de los pacientes fue similar, lo que demuestra que los factores de riesgo no están relacionados con el tipo de asistencia pública o privada, excepto en relación con el grupo de edad y la enfermedad asociada. Destacamos la alta incidencia de muerte encontrada en la población estudiada, lo que demuestra la necesidad de ampliar la atención preventiva.

Palabras clave: Factores de riesgo; Lesión por presión; Hospitales.

\section{Introdução}

Com o avanço da ciência e da tecnologia, há uma porcentagem maior de sobreviventes por trauma e doenças crônicas, em consequência, os hospitais atendem indivíduos cada vez mais críticos e propensos às complicações, dentre elas o surgimento de Lesões por Pressão (LP) (Souza et al., 2006; Matos et al., 2010; Miyazaki et al., 2010; Silva et al., 2010).

Úlcera por pressão, úlcera de decúbito ou escaras foram alguns dos termos utilizados para definir a LP. O National Pressure Injury Advisory Panel (NPIAP) anunciou oficialmente, em 13 de abril de 2016, a mudança na terminologia Úlcera por Pressão para Lesão por Pressão no intuito de esclarecer melhor a condição e facilitar a notificação da mesma (NPIAP, 2016).

Esse tipo de condição pode aparecer a partir da intensidade e a duração da pressão exercida sobre a proeminência óssea e a superfície. Ela tem elevada incidência em pacientes hospitalizados, gerando custos emocionais e financeiros, para o paciente, para a família, para o hospital e para instituições de saúde, além de aumentar as taxas de morbidade e mortalidade, por isso é considerado um importante problema de saúde pública (Souza et al., 2006; Matos et al., 2010; Silva et al., 2010; Miyazaki et al., 2010).

Pode-se dividir os fatores de risco que facilitam o desenvolvimento das LP em dois tipos: os fatores extrínsecos e intrínsecos. Aqueles que agem diretamente nos tecidos, são os chamados fatores extrínsecos, estes independem do paciente. Por exemplo, a pressão local, o cisalhamento, a fricção local e a umidade (Luz et al., 2010; Marini, 2016).

Aqueles fatores que são relativos ao indivíduo, isto é, aqueles que se referem ao estado físico do paciente, são os considerados fatores de risco intrínsecos. Os principais elementos encontrados nesta classificação são imobilidade, presença de incontinência urinária e/ou fecal, alterações na perfusão sanguínea da pele e presença de doenças neurológicas (NPIAP, 2020; Luz et al., 2010). Além destes, são citados por outros autores a desnutrição (Serpa \& Santos, 2008) e a senescência cutânea (alterações fisiológicas e cutâneas inerentes ao envelhecimento) (Abuchaim et al., 2010) como fatores de vulnerabilidade para 
surgimento e agravamento das LP. Os estudos acerca das LP apresentam dados com uma vasta alternância, decorrentes de metodologias diferentes e varia de acordo com o tipo de população estudada (Luz et al., 2010; Marini, 2016).

Sendo assim, faz-se necessário buscar, revelar e vincular evidências na saúde das populações como resultado de fatores contextuais da vida. Como a LP é problema de saúde pública, ou seja, de grande expressão, a investigação dos fatores intrínsecos e extrínsecos são fundamentais (Mata \& Costa, 2019).

Esta condição de saúde exige a continuidade e o prolongamento do cuidado, que não termina com a hospitalização, gerando mais gastos para a família no atendimento domiciliar (Miyazaki et al., 2010; Pessoa et al., 2011; Mata \& Costa, 2019).

A escassez, em âmbito nacional, de diretrizes que orientem a prevenção e o tratamento da LP e a necessidade de utilizar as diretrizes internacionais para formular orientações, sugestões para os planos para saúde brasileira, explica porque os autores reforçam a necessidade de aumentar o conhecimento científico da LP. Infelizmente, atualmente, o que encontramos na prática clínica, não são condutas baseadas em evidências, e sim mitos, tradições e experiências próprias ou de colegas (Souza \& Santos, 2007; Miyazaki et al., 2010; Souza et al., 2017).

Sabe-se que quando ofertada assistência de qualidade, as consequências da LP podem ser minimizadas e o processo curativo mais efetivo. O conhecimento dos fatores predisponentes, individuais e institucionais, para o surgimento da LP podem influenciar na tomada de decisão dos profissionais quanto a melhor estratégia para sua prevenção e tratamento, fomentando a importância desse estudo que objetivou conhecer o perfil clínico e epidemiológico de pacientes que adquiriram LP durante o período de internamento hospitalar, comparando as características entre o hospital público e o privado.

\section{Metodologia}

Trata-se de um estudo analítico, comparativo retrospectivo, de abordagem quantitativa, o qual analisou as variáveis por meio da leitura de prontuários autorizados pela direção dos hospitais. A coleta foi iniciada após aprovação do comitê de ética e pesquisa da Universidade Estatual de Ciências da Saúde, sob o parecer n ${ }^{\circ}$ 2.144.756, e autorização dos hospitais envolvidos.

Os prontuários inclusos foram dos pacientes adultos, com descrição de presença de LP, adquirida durante o internamento hospitalar, no período de corte delimitado pelo pesquisador, que resulta no total de três meses em cada hospital realizado no ano de 2018. A amostra foi dependente da quantidade de pacientes cuja descrição de obtenção de LP estava descrita no prontuário nesse período de tempo.

Foram excluídos os prontuários ilegíveis, de pacientes menores de 18 anos e os que foram transferidos de outro hospital com o tempo superior a 30 dias ou que adquiriram LP fora desse processo hospitalar.

As variáveis foram coletadas nos prontuários manualmente, através do preenchimento de um formulário de coleta de dados, elaborado pelos pesquisadores, sendo elas: sexo, faixa etária, grau de escolaridade, índice de massa corporal (IMC), tempo de internação (curto (<1mês), médio (1 a 6 meses) e longo (>6meses), doenças associadas, óbito, acompanhamento fisioterapêutico, atendimento multidisciplinar, local e tratamento da LP.

No hospital público foram analisados os prontuários arquivados no Serviço de Arquivo Médico do Hospital (SAME) de forma retrospectiva. Nesse setor, são arquivados os prontuários dentro de pastas numeradas e organizadas por datas, em prateleiras separadas por anos. Todos os prontuários deste período foram avaliados, inicialmente em uma etapa de triagem em busca dos prontuários dos pacientes que se enquadraram no critério de inclusão. Posteriormente foram preenchidos os formulários de coletas de dados com as informações dos pacientes inclusos.

No hospital privado, a coleta foi efetuada nos prontuários arquivados no banco de dados do hospital. Nesse local, o acesso aos dados ocorreu por meio de um sistema acessado via rede de internet e um aparelho eletrônico visual como computador/notebook. Ademais, assim como no hospital público, foram coletados os dados dos prontuários inclusos e 
preenchido o formulário de coleta de dados com as variáveis selecionadas. Utilizou-se os seguintes filtros para realização da triagem dos dados: período de internamento, idade e a descrição de LP adquirida durante o internamento hospitalar. Foi observada a ausência de informação neste sistema quanto ao tratamento utilizado na LP e para colher esta informação foi realizado contato verbal com a equipe de enfermagem, responsável pelo tratamento e notificação das LP.

Finalizado o processo de coleta, iniciou-se o processo de análise dos dados, utilizando-se o programa Microsoft Office Excel, por meio de estatística descritiva, calculando-se a média das variáveis numéricas e as frequências relativas e absolutas das variáveis categóricas. Os dados foram apresentados em gráficos e tabelas.

\section{Resultados}

Do hospital público, foram triados 2.202 prontuários, no período do estudo, destes, 65 (2,9\%) prontuários se enquadraram nos critérios de inclusão. Este foi o número total da amostra do hospital público. No hospital privado, foram triados 230 prontuários, no mesmo período, destes, 34 (14,7\%) correspondeu ao número total da amostra do hospital. Os outros 2.137 prontuários do hospital público não entraram na amostra pelos critérios: menores de 18 anos, os transferidos de outro hospital com o tempo superior a 30 dias e os que já apresentavam LP no dia da admissão hospitalar, os mesmos critérios foram os quais excluíram os 196 pacientes da amostra do hospital privado. Referente aos prontuários que se enquadraram nos critérios de inclusão, em nenhum dos hospitais houve prontuários ilegíveis e por isso manteve-se esse o número da amostra.

Quando analisados os dados dos hospitais, separadamente, obtivemos os respectivos perfis clínicos e epidemiológicos encontrados nas Figuras 1 e 2.

Figura 1. Descrição do perfil clínico dos pacientes de um hospital público de Alagoas, Brasil, 2018.

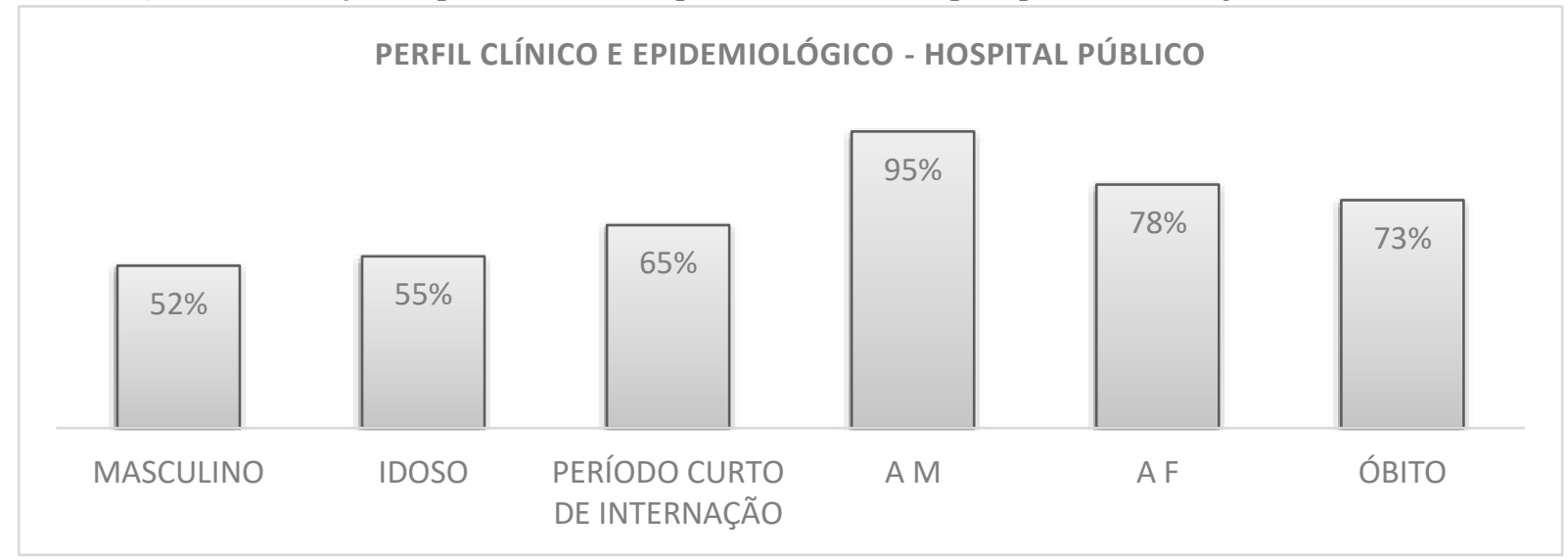

Legenda: $\mathrm{AM}=$ Atendimento Multiprofissional; $\mathrm{AF}=$ Atendimento Fisioterapêutico. Fonte: Autores.

Figura 2. Descrição do perfil clínico dos pacientes do hospital privado de Alagoas, Brasil, 2018.

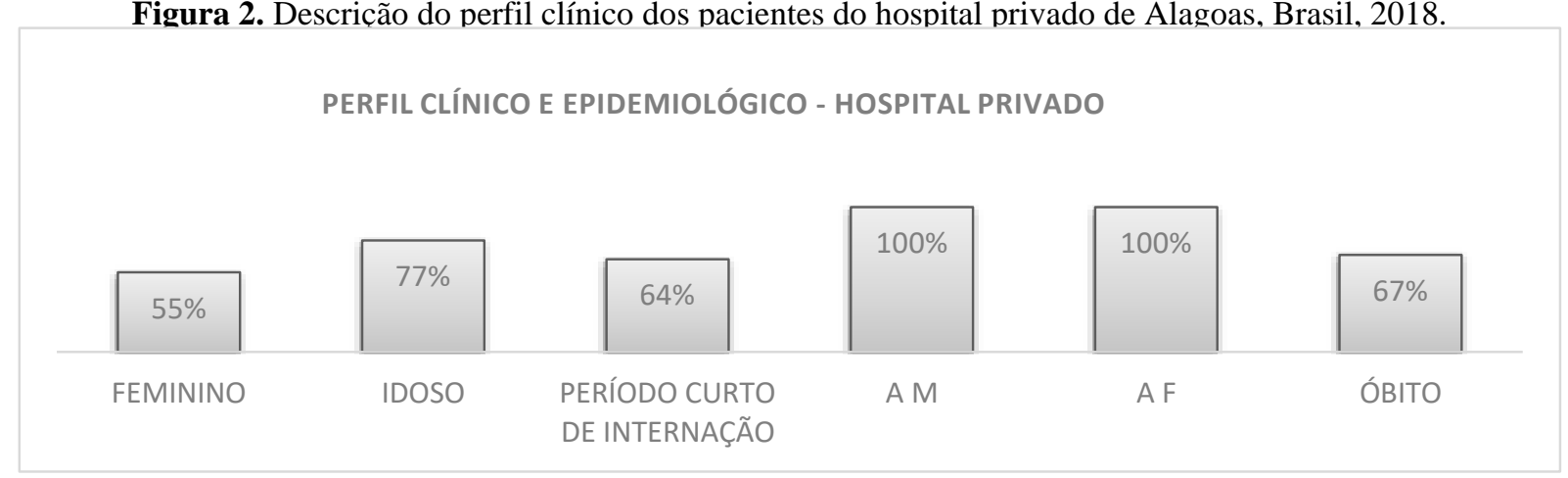

Legenda: $\mathrm{AM}=$ Atendimento Multiprofissional; $\mathrm{AF}=$ Atendimento Fisioterapêutico. Fonte: Autores . 
A Tabela 1 apresenta a frequência relativa e absoluta dos dados epidemiológicos dos pacientes diagnosticados com LP durante seu internamento, comparando os hospitais público e privado.

Tabela 1. Dados epidemiológicos dos pacientes diagnosticados com LP durante seu internamento, entre os hospitais público e privado. Alagoas, Brasil, 2018.

\begin{tabular}{|c|c|c|c|c|}
\hline \multirow{2}{*}{$\begin{array}{c}\text { VARIÁVEL } \\
\text { Sexo }\end{array}$} & \multicolumn{2}{|c|}{ HOSPITAL PÚBLICO } & \multicolumn{2}{|c|}{ HOSPITAL PRIVADO } \\
\hline & $\mathbf{N}$ & $\%$ & $\mathbf{N}$ & $\%$ \\
\hline Masculino & 34 & 52,31 & 15 & 45,86 \\
\hline Feminino & 31 & 47,69 & 18 & 55,14 \\
\hline \multicolumn{5}{|l|}{ Faixa Etária } \\
\hline Jovem & 9 & 14,00 & 2 & 7,00 \\
\hline Adultos & 20 & 31,62 & 5 & 17,10 \\
\hline Idosos & 36 & 55,38 & 23 & 77,90 \\
\hline \multicolumn{5}{|c|}{ Período de Internação } \\
\hline Curto & 42 & 64,62 & 21 & 64,14 \\
\hline Médio & 20 & 30,77 & 9 & 27,33 \\
\hline Longo & 3 & 4,62 & 3 & 9,52 \\
\hline \multicolumn{5}{|c|}{ Atendimento Multi } \\
\hline Sim & 62 & 95,38 & 33 & 100,00 \\
\hline Não & 3 & 4,62 & 0 & 00,00 \\
\hline \multicolumn{5}{|c|}{ Atendimento Fisio } \\
\hline Sim & 51 & 78,46 & 33 & 100,00 \\
\hline Não & 14 & 21,54 & 0 & 00,00 \\
\hline \multicolumn{5}{|l|}{ Óbito } \\
\hline Sim & 45 & 69,23 & 22 & 67,48 \\
\hline Não & 20 & 30,77 & 11 & 33,52 \\
\hline
\end{tabular}

Legenda: $\mathrm{N}=$ número da amostra; $\%=$ porcentagem; Multi = Multidisciplinar; Fisio = Fisioterapêutico. Fonte: Autores.

Observa-se que quando comparados os dados dos dois hospitais com pacientes que adquiriram LP neste ambiente, a relação percentual numérica entre homens e mulheres são muito semelhantes, assim como a faixa etária, predominantemente de idosos, conferida a pessoas que tem idade igual ou superior a 60 anos. Quanto ao período de internação, prevalece o número percentual de pacientes que passaram um tempo inferior a um mês internado no hospital, sendo esse um período considerado curto de internamento. No tocante a assistência profissional, encontramos pacientes que receberam atendimento multidisciplinar, ou seja, assistência de profissionais da saúde superior a duas especialidades na maioria dos pacientes, assim como o atendimento fisioterapêutico. Observou-se que o prognóstico mais frequente foi o óbito.

A Figura 3 apresenta o perfil clínico e epidemiológico dos pacientes que adquiriram lesão por pressão durante o internamento hospitalar unindo os hospitais público e privado, com os seguintes resultados: $50 \%$ feminino e 50\% masculino; idoso (62\%); com período de internação curto <1 mês (64\%), que receberam atendimento multidisciplinar (97\%) e fisioterapêutico (86\%) e foram a óbito $(68 \%)$. 
Figura 3. Descrição do perfil clínico dos pacientes dos hospitais público e privado de Alagoas, Brasil, 2018.

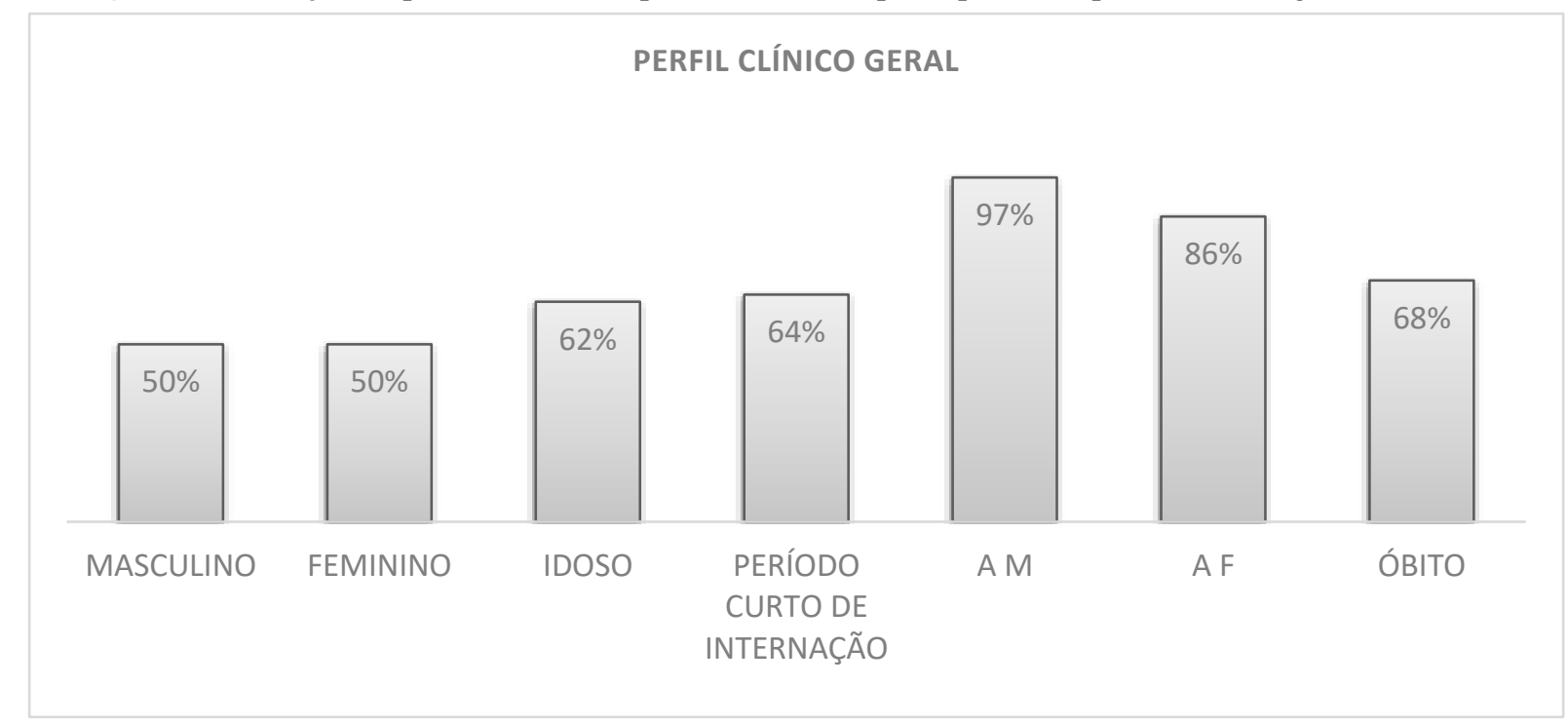

Legenda: $\mathrm{AM}=$ Atendimento Multiprofissional; $\mathrm{AF}=$ Atendimento Fisioterapêutico. Fonte: Autores .

Quanto ao tratamento da LP, as evoluções do hospital público, descrevem que é realizado basicamente pelos técnicos de enfermagem com o uso do Ácido Graxo Essencial (AGE) (67\%), a papaína associada ao AGE (10\%), curativo com o AGE (3\%) e só em uma maior gravidade (a qual não é descrita o critério de avaliação) é que utilizam a cirurgia plástica com a técnica de debridamento (10\%) feita pelo cirurgião plástico.

Já no hospital privado, no contato verbal com a equipe de enfermagem, responsável pelo tratamento e notificação das LP, na sua íntegra, foi relatado que o tratamento é definido pelo estágio da LP I, II, III e IV, conforme descrito abaixo:

- Estágio I: aplicação de Allevyn (curativo a base de espuma) e atenção quanto a mudança de decúbito;

- Estágio II: curativo com AGE ou gaze de Rayon;

- Estágio III: curativo com Purilon + Rayon ou apenas Purilon;

- Estágio IV: técnica de Square, curativo com papaína ou debridamento de ferida.

Achados do estudo registram a região sacral (68\%) como sendo a localização do corpo onde houve o maior número de LP, levando em consideração o local de surgimento da primeira lesão (em casos de mais de uma) e único, porém esta região também foi a mais acometida em casos de surgimento secundário, em pacientes que obtiveram mais de uma lesão por pressão. Foi encontrado também calcâneo (6\%), glútea (8\%), dorso (2\%), trocantérica (12\%), inter glútea (3\%) e perna (1\%).

A Tabela 2 apresenta a descrição das doenças e hábitos descritos nos prontuários dos pacientes com diagnóstico de LP. Foi possível identificar a alta prevalência de pacientes com Hipertensão Arterial Sistêmica (HAS) isolada (32,35\%), além da alta prevalência da associação de diabetes e hipertensão (26,5\%) dentro do perfil epidemiológico do hospital privado. Já no hospital público a maior prevalência foi de diabetes isolada $(15,15 \%)$. Acredita-se que essas comorbidades possam estar associadas a fragilidade da pele dos pacientes, no caso da diabetes mellitus, que acontece dificuldade na cicatrização de feridas por baixa perfusão de oxigênio e nutrientes (Andrade et al., 2013) e na HAS que altera a circulação cutânea e compromete o aporte de nutrientes e células de defesa dificultando a cicatrização de feridas (Rocha et al., 2020). 
Tabela 2. Descrição das doenças encontradas perfil clínico dos pacientes do hospital privado. Alagoas, Brasil, 2018.

\begin{tabular}{c|c|c}
\hline DOENÇAS/HÁBITOS & $\begin{array}{c}\text { HOSPITAL PRIVADO } \\
(\boldsymbol{\%})\end{array}$ & $\mathbf{( \% )}$ \\
\hline Diabetes e Hipertensão & 26,5 & 3,03 \\
Hipertensão & 32,35 & 7,6 \\
Diabetes & 11,77 & 15,15 \\
Tabagismo & 2,94 & 0 \\
Demência & 2,94 & 3,03 \\
AVC & 5,88 & 6,06 \\
Sem Patologias & 17,64 & 65,15 \\
\hline
\end{tabular}

Fonte: Autores.

A notificação de algumas informações referentes às condições clínicas do paciente foi negligenciada, como por exemplo: o índice de massa corporal ou mesmo a condição nutricional. Os poucos prontuários que apresentavam a informação, mostraram pacientes com baixo peso.

Outra informação não descrita nos prontuários hospitalares foi o grau de escolaridade, por este motivo, optou-se por buscar apenas a informação da alfabetização, que está presente no prontuário hospitalar. Porém, este também era negligenciado, dos 65 prontuários do hospital público, apenas 19 (29\%) tiveram esta informação descrita, sendo 17 alfabetizados e 02 analfabetos.

\section{Discussão}

Fazendo um comparativo entre os perfis clínico e epidemiológico dos hospitais, é possível identificar que a maioria das características dos pacientes dos dois hospitais foram muito semelhantes. As diferenças mais marcantes foram relacionadas às doenças/hábitos associadas a esses pacientes.

O perfil clínico do hospital privado nos mostra que os pacientes que adquiriram a LP possuíam uma doença associada, ou seja, presença de um fator não modificável associado à lesão. Já no hospital público é possível observar um perfil de pacientes predominantemente sem doenças associadas, demonstrando que o hospital público do estudo apresenta uma maior vulnerabilidade a fatores modificáveis (mobilidade no leito, hidratação da pele, estado nutricional), o que configura risco para uma quantidade maior de pacientes.

Na literatura é possível encontrar uma relação direta de comorbidades como doenças neurológicas, psíquicas ou que promovam alteração nos aspectos da pele com a presença da LP. É o caso da diabetes mellitus; doença presente nos pacientes do hospital privado deste estudo. Nestes casos o paciente diabético, apresenta comprometimento da perfusão sanguínea, o que acarreta em um inadequado fornecimento de oxigênio, nutrientes e antibióticos, para as células, em especial nos membros inferiores. Isso leva a uma disposição maior à hipoxemia dos tecidos, e assim uma facilidade no aparecimento de LP, como também à desorganização dos reparos teciduais, dificuldade de cicatrização das feridas, ocasionando atraso no processo de regeneração tecidual (Luz et al., 2010; Macedo et al., 2017).

Quanto à faixa etária, nosso estudo evidenciou em ambos os hospitais uma maior prevalência de LP em idosos. Tal fato se justifica pelas conhecidas consequências do envelhecimento do tecido cutâneo, tais como, a redução da espessura da pele, diminuição de fibras elásticas e a rigidez de colágeno, redução do tecido adiposo subcutâneo, diminuição de capilares da derme, 
Research, Society and Development, v. 10, n. 4, e18010413176, 2021

(CC BY 4.0) | ISSN 2525-3409 | DOI: http://dx.doi.org/10.33448/rsd-v10i4.13176

podendo gerar uma redução do suporte sanguíneo e desidratação da pele, tornando os idosos um grupo de indivíduos mais susceptíveis a desenvolver LP (Freitas et al., 2007; Fernandes et al., 2008).

Ainda referente às condições que podem propiciar o aparecimento dessa lesão, os dados nos mostram algumas situações diferentes da maioria da literatura, como por exemplo, o tempo de internação, em que estudos demonstram que a internação por tempo prolongado é o fator de risco mais importante para o aparecimento da LP, o que não é sustentado/corroborado pelo presente estudo (Santos et al., 2005; Pessoa et al., 2008)

O óbito foi um evento presente em $69 \%$ dos pacientes que adquiriram LP nos hospitais, resultado semelhante foi encontrado por Barbosa et al., (2019) ao realizar estudo em Fortaleza com 160 prontuários e destes, 55,6\% evoluíram com este desfecho. Esses achados corroboram com os dados da literatura em que a presença de LP aumenta as taxas de morbidade e mortalidade da população, enfatizando a relevância do estudo (Matos et al., 2010; Miyazaki et al., 2010; Rocha et al., 2006).

$\mathrm{O}$ tratamento da LP foi realizado pela equipe de enfermagem, sendo o AGE o principal tratamento descrito. A literatura nos mostra que o tratamento da LP é realizado por etapas, a primeira trata-se de uma análise das condições clínicas do paciente e da lesão e esta avaliação deve acontecer diariamente, observando dimensões, presença de tecido necrótico, tecido de granulação e por último, mas não menos importante: a evolução do plano analgésico e a presença de efeitos adversos, alérgicos ou complicações como infecção. Todo este processo precisa ser documentado e existem escalas de cicatrização, corroborando com as informações da equipe de enfermagem do hospital privado (Rangel \& Caliri, 2009; Duarte \& Alves, 2013).

A higiene das lesões deve ser realizada apenas com soro fisiológico e técnicas apropriadas de esterilização. Para escolher o curativo mais adequado para o tratamento das lesões por pressão, é necessário levar em conta a presença de tecido infectado, a quantia de exsudato, o grau de ressecamento da lesão e se há tecido necrótico. Na literatura atualmente, não existe opções de curativos que se sobressaiam significativamente sobre a outra, apesar de existirem várias opções como gazes, filme transparente, hidrocolóide, hidrogel, espuma de poliuretano, alginatos, carvão ativado com prata, colágeno e fatores de crescimento (Borba, 2015). A importância do debridamento do tecido necrótico se dá na interferência da cicatrização e na prevenção da infecção secundária (Rangel \& Caliri, 2009; Duarte \& Alves, 2013).

O pior progresso e complicação das lesões por pressão é a infecção, onde a apresentação clínica pode se dar por calor local, vermelhidão, exsudato e odor fétido; porém é possível encontrar diversas manifestações, sendo a demora na cicatrização da lesão o único sinal da infecção (Rangel \& Caliri, 2009; Duarte \& Alves, 2013).

É observado no estudo de Santos et al., 2019 e em outros da literatura que a equipe multidisciplinar tem papel importante no cuidado dos paciente com LP e na tomada de decisões com relação a prevenção e tratamento. No entanto, $\quad 97 \%$ dos pacientes que adquiriram LP receberam o atendimento multiprofissional, configurando assim o insucesso deste modelo de trabalho na prevenção e tratamento da LP neste estudo. Este dado nos faz refletir sobre a necessidade de otimizar e fortalecer o trabalho da equipe interdisciplinar para a prevenção e o tratamento da LP. É necessário capacitar nossos profissionais, desvincular o tratamento desse tipo de paciente apenas ao enfermeiro e do técnico de enfermagem, incluindo o fisioterapeuta e outros profissionais, tornando, assim, o tratamento interdisciplinar mais efetivo.

O imobilismo por tempo prolongado é um fator primário determinante para o aparecimento da LP. Entretanto, $86 \%$ dos pacientes que adquiriram LP recebiam o atendimento fisioterapêutico direcionado a cinesioterapia o que propiciava movimento ao corpo (Luz et al., 2010; Furieri et al., 2015).

Pode-se perceber a necessidade da capacitação fisioterapêutica na prevenção da LP e no tratamento da mesma, pois já existem evidências científicas quanto ao uso do gerador de alta frequência com resultados satisfatórios na regressão dos estágios das lesões por pressão, principalmente quanto ao efeito bactericida e/ou fungicida, que são as graves complicações das LP quando relacionadas ao óbito. (Say et al., 2003; Prado et al., 2017) 
Ressalta-se que apesar da grande quantidade de trabalhos científicos brasileiros relacionados a lesão por pressão, ainda não existem diretrizes nacionais para o tratamento da LP. Como consequência desta realidade, pode-se observar que os hospitais ainda realizam práticas do atendimento das LP não baseadas em evidências, e sim em mitos, tradições e experiências próprias ou de colegas (Miyazaki et al., 2010; Souza et al., 2017).

Mesmo havendo atendimento nutricional nos dois hospitais pesquisados não foram encontrados dados relacionados à sua condição nutricional e seu IMC nos prontuários, não sendo possível, assim, designar se o perfil clínico desses pacientes era desnutrido (baixo peso), nutrido (normal) ou obeso (peso elevado), fator esse que pode interferir no aparecimento da lesão. Os poucos prontuários que apresentavam a informação, mostraram pacientes com baixo peso.

Esta informação condiz com o que encontramos na literatura. $\mathrm{O}$ estado nutricional deficiente do paciente está diretamente relacionado com o desenvolvimento de LP e alerta para a importância de identificar o estado nutricional do paciente de forma precoce, pois desta maneira é possível reconhecer o paciente de risco e programar estratégias que previnam o surgimento dessas lesões. Estudos confirmam a eficiência da prevenção da desnutrição na redução do risco de LP ( Lourenço et al., 2012).

Ainda que a realidade na literatura seja de que a desigualdade social se constitua interesse da saúde pública e, assim sendo, passam a ser uma prioridade epidemiológica, repete-se a dificuldade na coleta de dados importantes como a condição nutricional e a escolaridade da amostra pesquisada. Não é visto correlação direta do nível de alfabetização e a LP na literatura, no entanto, estudos confirmam as influências da escolaridade e outros determinantes que podem ser medidos no nível individual, em realidades sobre a saúde das pessoas (Mata \& Costa, 2019).

É possível encontrar estudos que relacionam o analfabetismo à faixa etária do idoso, mas os resultados encontrados vão contra esta afirmativa. Além disso, estudos mostram a utilização de métodos didáticos e lúdicos como forte potencial de agradar, entusiasmar e ensinar ao auto cuidado, por exemplo, e sua direta eficiência com pacientes alfabetizados que possuem um melhor entendimento (Sokem et al., 2018; Diccini et al., 2009).

Foi possível perceber que há uma incidência de LP muito superior no hospital privado (14,7\%) quando comparado ao hospital público $(2,9 \%)$. Porém, fica o questionamento, se esta diferença é devido ao grande número e a variedade de paciente internos no hospital público, quando comparado ao hospital privado. Outro questionamento é quanto a efetividade na notificação, onde a equipe profissional do hospital privado pode estar mais atenta a notificação de LP do que a equipe do hospital público e, assim, haver alguma negligência na notificação. A metodologia deste estudo não foi capaz de responder a essas questões, apenas apontá-las. Sugerimos que outros estudos sejam realizados no intuito de abordar esse tema com essa ênfase.

\section{Considerações Finais}

O perfil clínico e epidemiológico dos hospitais público e privado foi semelhante nos itens sexo, faixa etária, local da lesão por pressão, atendimento fisioterapêutico, atendimento multidisciplinar, óbito e diferiram em relação às doenças associadas ao paciente. Pode-se concluir que a maioria das variáveis do perfil epidemiológico dos pacientes portadores de LP, dos dois hospitais, foi semelhante, corroborando com os dados encontrados na literatura sobre os pacientes com essa lesão.

A possível subnotificação da LP foi um viés nesta pesquisa, podendo interferir nos seus resultados finais. Além disso, a descrição da condição nutricional e do tratamento, também foram informações deficientes que alertaram os pesquisadores para a importância de ampliar estudos sobre esse tema.

Acredita-se na necessidade de capacitar a equipe para avaliação detalhada e notificação da LP, reforçando a necessidade da utilização de escalas que avaliem a condição e o progresso destas complicações, além de treiná-los para a utilização de tratamentos baseados em evidências e na interdisciplinaridade, ampliando os recursos terapêuticos e inserindo a Fisioterapia no 
tratamento. Tais medidas poderão reduzir o tempo de cicatrização de lesão, diminuindo o tempo de internação e os custos com o tratamento, além de diminuir as chances de infecções que são a principal causa da elevada taxa de mortalidade associada a esta condição.

\section{Referências}

Abuchaim, S., Eidt, L. M., \& Viegas, K. Úlceras por pressão, nutrição e envelhecimento cutâneo. In: Schwanke, C. H. A., Gomes, I., Antunes, M. T., \& Closs, V. E. (2010). Atualizações em Geriatria e Gerontologia III nutrição e envelhecimento (3a ed): Editora EdiPUCRS, 312p.

Andrade, M. G. L., Camelo, C. N., Carneiro, J. A., \& Terêncio, K. P. (2013). Evidências de alterações do processo de cicatrização de queimaduras em indivíduos diabéticos: revisão bibliográfica. Revista Brasileira de Queimaduras, 12(1), 42-48.

Barbosa, A. S., Oliveira, E. S., Leite, M. G., Feitosa, D. S. L. L., Studart, L. M. B., Cavalcante, T. M. C., \& Oliveira, P. M. P. (2019). Perfil clínico dos pacientes acometidos por lesão por pressão. Revista Enfermagem Atual in Derme, 88(26), 01-09.

Bezerra, S. M. G. (2010). Prevalência de úlceras por pressão em pacientes acamados e cuidados dispensados em domicílio (Dissertação de mestrado) Universidade Federal do Piauí, Teresina, PI, Brasil. https://www.revistaestima.com.br/index.php/estima/article/view/336.

Borba, P. N. (2018). Tipos de curativos utilizados durante o tratamento da úlcera por pressão. Revista Científica Semana Acadêmica, 124(1), 1-38.

Diccini, S., Camaduro, C., \& Lida, L. I. S. (2009). Incidência de úlcera por pressão em pacientes neurocirúrgicos de hospital universitário. Acta Paulista de Enfermagem, 22(2), 205-209.

Duarte, A. V., \& Alves, M. A. (2013). Úlcera de pressão: fatores de risco em pacientes hospitalizados. Revista Diálogos Acadêmicos, 2(2), 102-108.

Fernandes, L. M., Caliri, M. H. L., \& Haas, V. J. (2008). The effect of educative interventions on the pressure ulcer prevention knowledge of nursing professionals. Acta Paulista de Enfermagem; 21(2), 305-311.

Freitas, K. S., Kimura, M., \& Ferreira, K. A. S. L. (2007). Necessidades de familiares de pacientes em unidades de terapia intensiva: Análise comparativa entre hospital público e privado. Revista Latino-americana Enfermagem; 15(1), 84-92.

Furieri, F. P. M., Uessugui, H. M., Oliveira, R. R., \& Fagundes, D. S. (2015). Atuação fisioterapêutica na úlcera por pressão: uma revisão. Revista Científica da Faculdade de Educação e Meio Ambiente, 6, 69-80.

Lourenço, T. M., Lenardt, M. H., Kletemberg, D. F., Seima, M. D., Tallmann, A. E. C., \& Neu, D. K. M. (2012). Capacidade funcional no idoso longevo: uma revisão integrativa. Revista Gaúcha de Enfermagem, 33(2), 176-185.

Luz, S. R., Lopacinski, A. C., Fraga, R., \& Andrade, C. (2010). Úlceras de pressão. Revista Brasileira Geriatria \& Gerontologia; 4(1), 36-43.

Macedo, J. L., Pereira, I. C., Oliveira, A. S. S., \& Magalhães, M. J. S. (2017). Eficácia da fitoterapia no processo de cicatrização tecidual de pacientes com diagnóstico de diabetes mellitus. Revista Ciência \& Saberes - Facema, 3(1), 396-400.

Marini, M. F. V. (2016). Úlceras de Pressão. In: Freitas, E.V., Py. L., Cançado, F., Doll, J., \& Gorgoni, M. L. Tratado de geriatria e gerontologia: GuanabaraKoogan, 981-991.

Mata, M. S., \& Costa, I. C. C. (2019). Composição do índice de iniquidade em saúde a partir das desigualdades na mortalidade e condições socioeconômicas em uma capital Brasileira. Revista Ciência \& Saúde Coletiva, 25(5), 1629-1640.

Matos, L. S., Duarte, N. L. V., \& Minetto, R. C. (2010). Incidência e prevalência de úlcera por pressão no CTI de um hospital Público do DF. Revista Eletrônica de Enfermagem, 12(4), 719-726.

Miyazaki, M. Y., Caliri, M. H. L., \& Santos, C. B. (2010). Conhecimento dos profissionais de enfermagem sobre prevenção da úlcera por pressão. Revista Latino-Americana de Enfermagem, 18(6), 1-10.

National Pressure Injury Advisory Panel (NPIAP). https://npiap.com/page/PressureInjuryStages.

Pessoa, E., Rocha, J., \& Bezerra, S. (2011) Prevalência de úlcera por pressão em pacientes acamados, cadastrados na Estratégia de Saúde da Família: um estudo de enfermagem (tese). Universidade Federal do Piauí, Teresina, PI, Brasil. https://revistainterdisciplinar.uninovafapi. edu.br/revistainterdisciplinar/v4n1/pesquisa/p2_v4n1.pdf.

Prado, Y. S., Tiengo, A., \& Brasil, A. C. (2017). A influência do estado nutricional no desenvolvimento de lesões por pressão em pacientes suplementados. RBONE - Revista Brasileira de Obesidade, Nutrição e Emagrecimento, 11(68), 699-709.

Rocha, J. A., Miranda, M. J., \& Andrade, M. J. (2006). Abc, tratamento da úlcera por pressão por enfermeiros de um hospital geral. Revista Eletrônica de Enfermagem, 11(1), 70-77.

Rocha, S. S., Pontes, E. D. S., Rocha, S. R. S., \& Falcone, A. P. M. (2020). Análise da presença de lesão por pressão em pacientes hospitalizados e as principais comorbidades associadas. Reserch Society and Development, 9(4), 1-14.

Santos, V. L. C. G., Azevedo, M. A. J., Silva, T. S., Carvalho, V. M. J., \& Carvalho V. F. (2005). Adaptação transcultural do pressre ulcer scale for healing (PUSH) para a língua portuguesa. Revista Latino-Americana de Enfermagem, 13(3), 305-313. 
Research, Society and Development, v. 10, n. 4, e18010413176, 2021

(CC BY 4.0) | ISSN 2525-3409 | DOI: http://dx.doi.org/10.33448/rsd-v10i4.13176

Santos, M. L., Silva, A. M. M., Vinagre, L. M F., Júnior, J. N. B. S., Miranda, Y. A. S., Silva, C. R. R., Brito, F. M. B., \& Anísio, B. K. F. (2019). Cicatrização de lesão por pressão: relato de caso. Revista de enfermagem UFPE on line, 13, 1-7.

Say, K. G., Gonçalves, R. C., Rennó, A. C. M., \& Parizotto, N. A. (2003). O tratamento fisioterapêutico de úlceras cutâneas venosas crônicas através da laserterapia com dois comprimentos de onda. Revista Fisioterapia Brasil, 4(1), 40-49.

Serpa, L. F., \& Santos, V. L. C. G. (2008). Desnutrição como fator de risco para o desenvolvimento de úlcera por pressão. Revista Acta Paulista de Enfermagem, 21(2), 367-369.

Silva, E. W. N. L., Araújo, R. A., Oliveira, E. C., \& Falcão, V. T. F. L. (2010). Aplicabilidade do protocolo de prevenção de úlcera de pressão em unidade de terapia intensiva. Revista Brasileira de Terapia Intensiva, 22(2), 175-185.

Sokem, J. A. S., Bergamaschi, F. P. R., \& Watanabe, E. A. M. T. (2018). O uso do jogo como ferramenta pedagógica para o ensino sobre dermatite associada à incontinência em ambiente hospitalar. Anais do Simpósio Ibero-Americano de Tecnologias Educacionais, $362-369$.

Souza, C. A., Santos, L., \& Silva, L. D. (2006). Aplicando recomendações da Escala de Braden e prevenindo úlceras por pressão - evidências do cuidar em enfermagem. Revista Brasileira de Enfermagem, 59(3), 279-284.

Souza, D. M. S. T., \& Santos, V. L. C. G. (2007). Risk factors for pressure ulcer development in institutionalized elderly. Revista Latino-Americana de Enfermagem, 15(5), 958-964

Souza, N. R., Freire, D. A., Souza, M. A. O., Melo, J. T. S., Santos, L. V., \& Bushatsky, M. (2017). Fatores predisponentes para o desenvolvimento da lesão por pressão em pacientes idosos: uma revisão integrativa. Revista Estima, 15(4), 229-239. 